\title{
Is a Picture Worth a Thousand Words? Effectiveness of iPad Technology in Preclinical Dental Laboratory Courses
}

\begin{abstract}
Michele L. Kirkup, Brooke N. Adams, Paul E. Reifeis, Jeni L. Heselbarth, Lisa H. Willis
Abstract: Dental educators should consider alternative modalities of instruction when experiencing difficulties conveying feedback to students. The aim of this study was to determine if integrating iPad technology as a visual learning tool would enhance the exchange of assessment information and improve academic performance in Indiana University School of Dentistry's preclinical curriculum. In 2016, the first-year Tooth Morphology (TM) and second-year Fixed Prosthodontics (Fixed) courses implemented a project using iPad images that allowed instructors to annotate acceptable and deficient areas of students' tooth wax-ups and preparations. In the two courses, all students (TM n=106 and Fixed n=105) and instructors (TM $n=21$ and Fixed n=17) were given pre-intervention surveys to report their perceived effectiveness of verbal feedback and were given post-intervention surveys to rate their experiences with iPad image feedback. Response rates for students in the two courses on the pre surveys were TM $87.7 \%$ and Fixed $85.7 \%$ and on the post surveys were TM $26.4 \%$ and Fixed $76.2 \%$. Response rates for instructors on the pre surveys were TM $52.4 \%$ and Fixed $82.4 \%$ and on the post surveys were TM $76.2 \%$ and Fixed $76.5 \%$. The results showed that a majority of both groups preferred the combination of verbal and iPad image feedback: $53 \%$ of responding students in TM and $51 \%$ in Fixed, and $75 \%$ of instructors in TM and 77\% in Fixed. In the TM course, responding instructors had a statistically significantly higher agreement than students that feedback with iPad images was superior to verbal feedback alone $(p=0.008)$. Furthermore, a multi-year analysis of TM practical examination grades found statistically significant lower change scores for the first and second exams in 2014 and 2015 compared to the 2016 scores when the iPad intervention occurred. These results suggest that verbal feedback combined with iPad images resulted in an enhanced exchange of information and increased student grades, particularly in the first-year dental curriculum.
\end{abstract}

Michele L. Kirkup, DDS, is Clinical Assistant Professor, Department of Prosthodontics, Indiana University School of Dentistry; Brooke N. Adams, DDS, is Clinical Assistant Professor, Cariology, Operative Dentistry, and Public Health Department, Indiana University School of Dentistry; Paul E. Reifeis, DDS, JD, MSD, is Clinical Assistant Professor, Cariology, Operative Dentistry, and Public Health Department, Indiana University School of Dentistry; Jeni L. Heselbarth, DDS, was a dental student at Indiana University School of Dentistry at the time of this study; and Lisa H. Willis, DDS, MSD, is Clinical Assistant Professor, Cariology, Operative Dentistry, and Public Health Department, Indiana University School of Dentistry. Direct correspondence to Dr. Michele Kirkup, Indiana University School of Dentistry, Department of Prosthodontics, 1121 West Michigan Street, Rm. 316 , Indianapolis, IN 46202; 317-274-5576; mkirkup@iu.edu.

Keywords: dental education, educational technology, instructional materials/methods, preclinical skills, iPad

Submitted for publication 7/16/18; accepted 10/12/18; first published online 2/11/19

doi: 10.21815/JDE.019.049

I $\mathrm{n}$ dental education, timely instructor feedback and detailed guidance are essential for preclinical students as they develop fine motor skills and selfassessment abilities. ${ }^{1}$ Dental students must develop these critical skills early in the educational process to aid in their professional growth and become confident in their clinical judgment., ${ }^{2,3}$ Specific feedback on students' performance is essential to the assessment process, as it allows the learner to identify strengths and weaknesses and to find mechanisms to address his or her learning gap. ${ }^{4}$ Educators often know what "acceptable" looks like based on the ideal concepts, but may have difficulty conveying these high-quality expectations to students. ${ }^{5}$ Preclinical instructors face a distinct challenge in explaining the criteria when assessing students' performance due to the confined environment of the procedures. When the standard of acceptability is measured in fractions of millimeters, instructors and students may find it difficult to pinpoint these minute variations. These challenges could lead to students' inability to identify critical inaccuracies, thus resulting in delayed development of self-evaluation skills.

Commission on Dental Accreditation (CODA) standards require dental education programs to use technology to "improve patient care, and to revolu- 
tionize all aspects of the curriculum, from didactic courses to clinical instruction" (p. 15). ${ }^{6}$ Recent innovations in computer-aided design/computer-aided manufacturing $(\mathrm{CAD} / \mathrm{CAM})$ and the development of digital evaluation software programs allow students to obtain feedback while viewing the results of their performance in a virtual environment. ${ }^{7}$ The instant feedback is given when students scan their preparations or tooth wax-ups with hand-held laser cameras. The scan is exported into an evaluation software program, which aligns the student's project with a master model and reports the variations. ${ }^{8}$

Although this evaluation tool has reported some success, digital comparing software and the supporting equipment also have limitations. The significant challenges for dental schools planning to invest in these expensive systems include the continued upgrades and the necessity of providing extensive training for both faculty and students to prevent user error. Other reported limitations are the process of precise alignment and technique-sensitive scanning, which may produce an inaccurate assessment. ${ }^{8,9}$ The Callan et al. study also reported that the students encountered difficulties during the scanning and, as a result, chose not to use this technology as an evaluation tool. ${ }^{9}$ In a study by Park et al. published in 2017, prepCheck software by Sirona Dental Systems (Long Island City, NY, USA) was used by preclinical students to evaluate tooth preparations. ${ }^{10}$ Although that study reported prepCheck was beneficial, the authors noted that improvements to the efficiency of the scanning process would enhance its user-friendliness as perceived by students.

Adopting mobile technology will be a necessity as higher education shifts to a more personalized student-centered learning model. A case study of graduate students found that over 1.5 million Apple iPad tablets (Cupertino, CA, USA) were used in educational settings, and more than 20,000 teaching applications were available to students. ${ }^{11}$ Compared to textbooks or traditional computers, one study found that tablet technology had the benefits of convenience, multiple applications, lower expense, and ease of use. ${ }^{12}$ Stein et al. argued that dental schools should embrace new technology trends and adapt teaching methods to promote student learning. ${ }^{13}$ There has been almost no research on the use of iPads in dental education to examine if they can improve students' performance in the preclinical setting. In the one study we found, instructor-led procedural videos that showed acceptable and unacceptable examples of tooth preparations were made available to students. ${ }^{14}$ That study concluded that using iPads as teaching tools in preclinical laboratories may offer students an enhanced learning environment and foster self-assessment.

In 2016, an iPad image feedback project was implemented in the preclinical laboratory courses at Indiana University School of Dentistry (IUSD). The purpose of this project was to enhance the effectiveness of preclinical laboratory teaching by using images taken with iPads, allowing instructors to annotate acceptable and deficient areas of students' tooth wax-ups and preparations from the instructor's perspective. Previous studies found that using visual learning tools, which provided students with specific instructions, significantly improved their performance. ${ }^{15,16}$ To support the use of visual resources, the Gadbury-Amyot et al. study explained how the Dual-Coding Theory can be applied to learning in dental education. ${ }^{14}$ This theory states that, by engaging both the visual and verbal processes when learning new material, the result is greater recall. ${ }^{14,17}$ Our project provided customized "live" visual assessment of student performance combined with instructors' verbal feedback. Furthermore, students received these images via sharing to a secure online platform to allow for reflective opportunities and documentation of their progression through the curriculum. The aim of this study was to determine if integrating iPad technology as a visual learning tool would enhance the exchange of assessment information and improve academic performance in IUSD's preclinical curriculum.

\section{Materials and Methods}

This study was determined to be exempt from oversight by the Indiana University Institutional Review Board (\#1606179055). The iPad feedback project was funded by a curriculum enhancement grant from the Center for Teaching and Learning at Indiana University-Purdue University Indianapolis for the 2016-17 academic year, and the authors were the project investigators. For this project, ten mini iPads were purchased for use as assessment tools in IUSD's preclinical laboratory courses: Tooth Morphology (TM) in the first-year DDS curriculum, and Fixed Prosthodontics (Fixed) in the second-year DDS curriculum. The free drawing application called Image Annotation (Panther Studio) and the online storage platform application Box (Redwood City, CA, USA) were loaded onto each iPad. The Image 
Annotation application allowed instructors to take images of tooth wax-ups or preparations, annotate them, and securely share the information with individual students via Box (Figure 1).

In August 2016, pre-intervention surveys were distributed to all students in the TM $(n=106)$ and Fixed $(n=105)$ courses to determine their perceived effectiveness of verbal feedback on their tooth waxups and preparations and their ability to recall the assessment information. A check item instrument with response options of strongly agree, agree, neither agree nor disagree, disagree, and strongly disagree was used. Pre-intervention surveys were also disseminated to all preclinical instructors in the TM course $(n=21)$ and Fixed course $(n=17)$ to collect their perspectives on the effectiveness of their verbal feedback to students and the students' ability to recall the feedback. A check item instrument with response options from strongly agree to strongly disagree was also used for this data collection.
Initially in each course, the instructors assessed students' performance using traditional verbal feedback. Following completion of the first practical exams in the TM and Fixed courses, the instructors participated in iPad feedback calibration sessions, which included training on annotating and sharing images to students' folders stored in the Box online platform. In September 2016, the instructors began taking iPad images to annotate students' projects when providing verbal feedback. Students were encouraged to review these images in Box for reflection opportunities.

After the intervention, post surveys were given to all the TM students $(\mathrm{n}=106)$ and Fixed students $(\mathrm{n}=105)$. The post surveys asked students to rate the effectiveness of the instructors' feedback using iPad images, their preferred instructor feedback method, and the helpfulness of referencing iPad feedback through Box. Similar check-item post surveys with the same subscales were given to all preclinical instructors (TM n=21 and Fixed $n=17$ ) at the conclusion of the
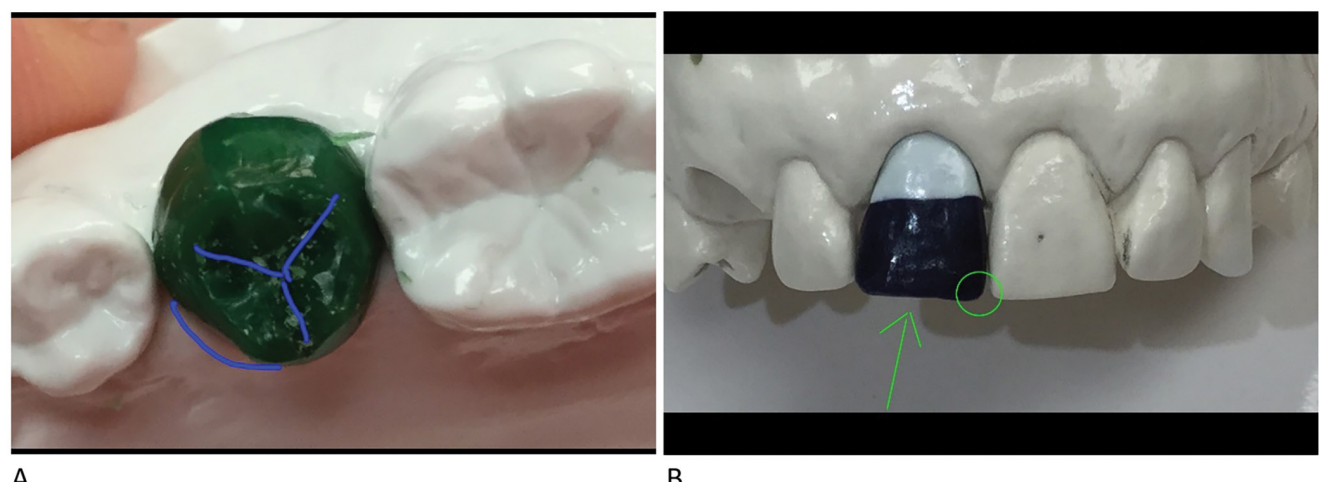

A

B
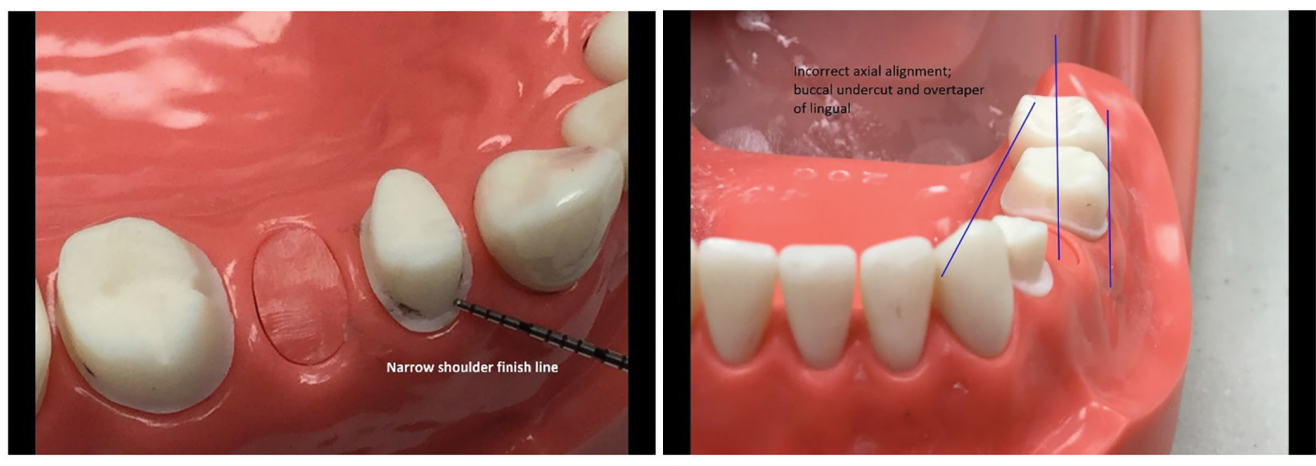

C

D

Figure 1. iPad images with instructor annotations

Note: Panel A is iPad image annotated to show deficiency in mesial contour and correct groove placement for premolar tooth wax-up. Panel B is iPad image annotated to highlight improper incisal edge and MIF point angle contour for central incisor wax-up. Panel C is iPad image annotated to demonstrate narrow shoulder finish line for metal ceramic crown preparation. Panel D is iPad image annotated to display incorrect axial alignment, buccal undercut, and overtaper of lingual surface for full gold crown preparation. 
iPad project (January 2017) to collect their responses on the effectiveness of conveying feedback through iPad images. Additionally, the instructors were asked to rate the user-friendliness of annotating and sharing images during student evaluation sessions and their preferred instructor feedback methods.

For the pre- and post-iPad intervention student grade analysis, we compared the TM and Fixed students' first practical exam mean scores from 2016 to the previous practical exam mean scores from 2014 and 2015 using the Wilcoxon Signed Rank Test, comparing pre- versus post-iPad intervention student scores. To determine if participating in iPad feedback in 2016 resulted in a significant difference in students' grades compared to the two previous years, the nonparametric Wilcoxon Rank Sum Test was applied for comparison between years. Additionally, a comparison analysis using Wilcoxon Rank Sum Test was performed to determine if TM and Fixed students who did reference the iPad images in Box achieved higher scores than students who did not reference Box.

\section{Results}

Response rates for students in the two courses on the pre-intervention surveys were TM $87.7 \%$ and Fixed $85.7 \%$ and on the post surveys were TM $26.4 \%$ and Fixed $76.2 \%$. Response rates for instructors on the pre surveys were TM $52.4 \%$ and Fixed $82.4 \%$ and on the post surveys were TM 76.2\% and Fixed 76.5\%.

\section{Pre-Intervention Survey}

To report the data collected on the five-point Likert scale, we combined strongly agree and agree to indicate a positive response and combined disagree and strongly disagree to indicate a negative response; neither agree nor disagree was a neutral response. The majority of TM student respondents $(83.9 \%)$ reported they could visualize verbal feedback from the instructor's perspective, but only $45.5 \%$ of the TM instructor respondents agreed that students could effectively visualize their verbal feedback. When asked about feedback recall ability, the majority of TM student (81.7\%) and instructor (72.7\%) respondents agreed that the students could recall the feedback provided (Figure 2).

In the Fixed course results, $72.2 \%$ of the student respondents agreed they could visualize the instructor feedback, 22.2\% gave a neutral response, and 5.6\% disagreed. For the Fixed instructor respondents, $14.3 \%$ gave a neutral response, and $35.7 \%$ disagreed that students could effectively visualize the instructor feedback. However, when asked about recalling the instructor feedback, large percentages of both Fixed student $(88.8 \%)$ and instructor $(92.8 \%)$ respondents agreed that students could recall the given feedback (Figure 3).

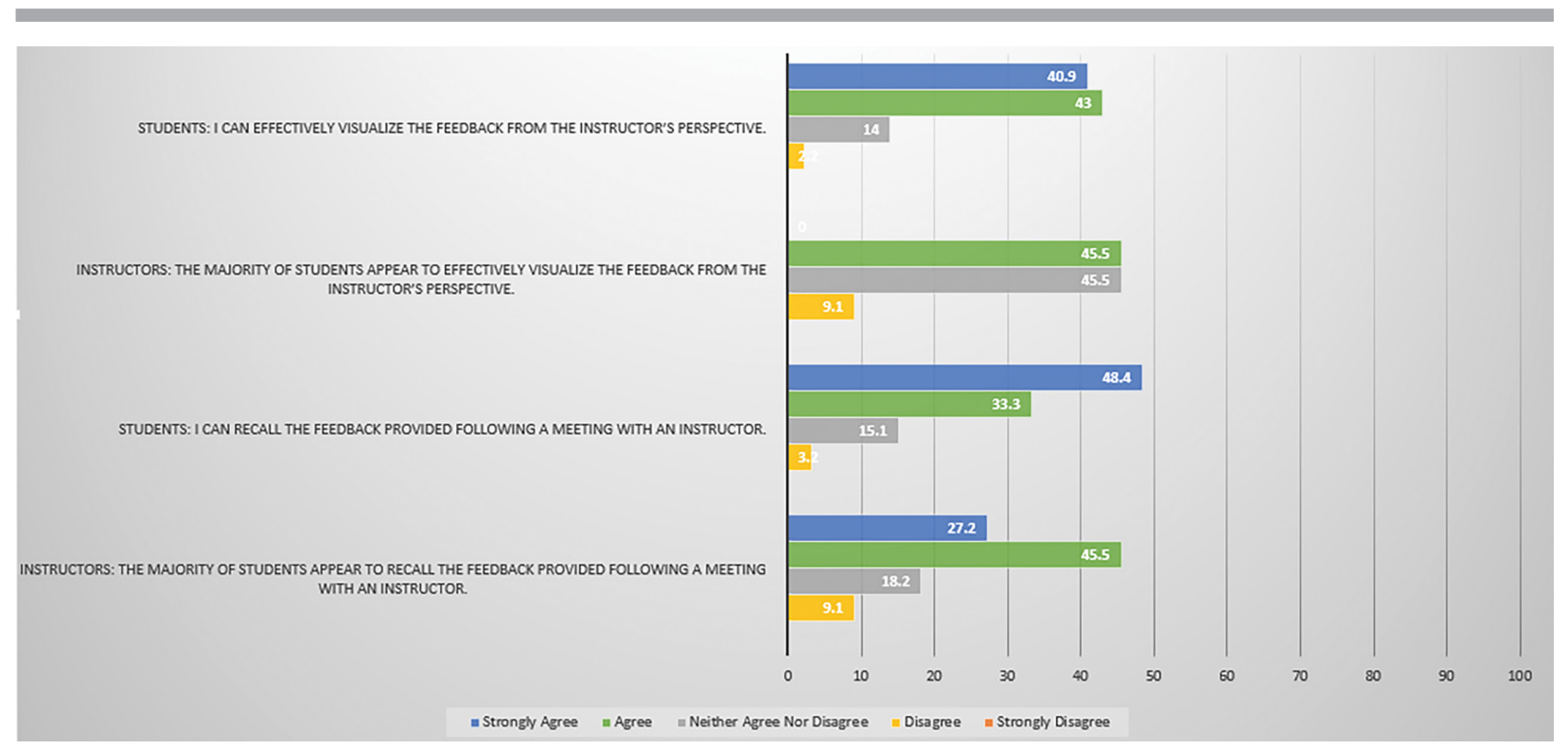

Figure 2. Results of pre survey in Tooth Morphology course, by percentage of responding students $(n=93)$ and instructors $(\mathbf{n}=\mathbf{1 1})$ 


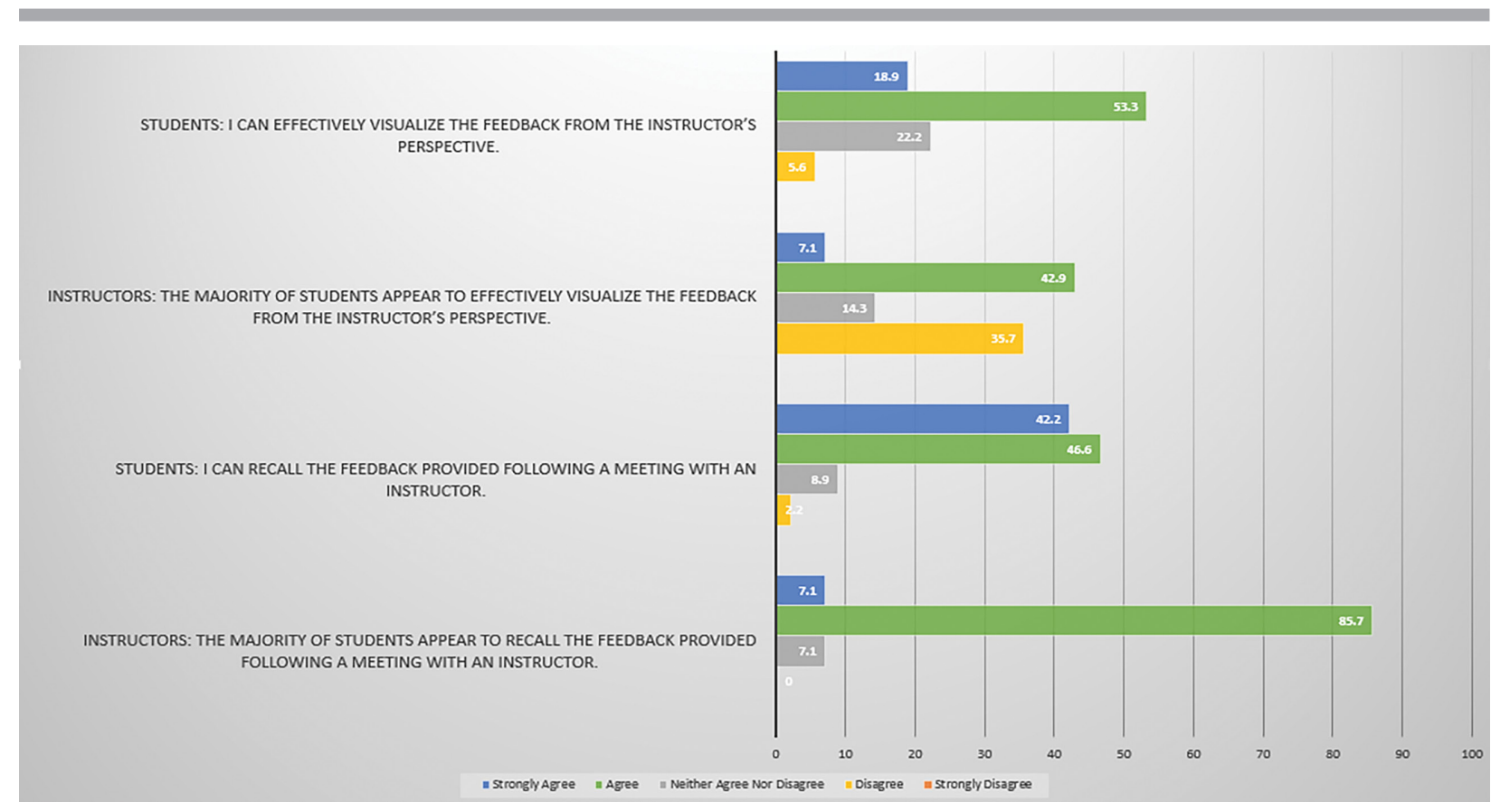

Figure 3. Results of pre survey in Fixed Prosthodontics course, by percentage of responding students $(\mathrm{n}=90)$ and instructors $(n=14)$

\section{Post-Intervention Survey}

On the post survey, $46 \%$ of TM student respondents and $81.3 \%$ of instructor respondents reported that evaluating tooth wax-ups using iPad feedback was very helpful or somewhat helpful (Figure 4). Furthermore, $62.2 \%$ of responding instructors found taking, annotating, and sharing iPad images during student evaluation sessions to be user-friendly or somewhat user-friendly. A statistically significant

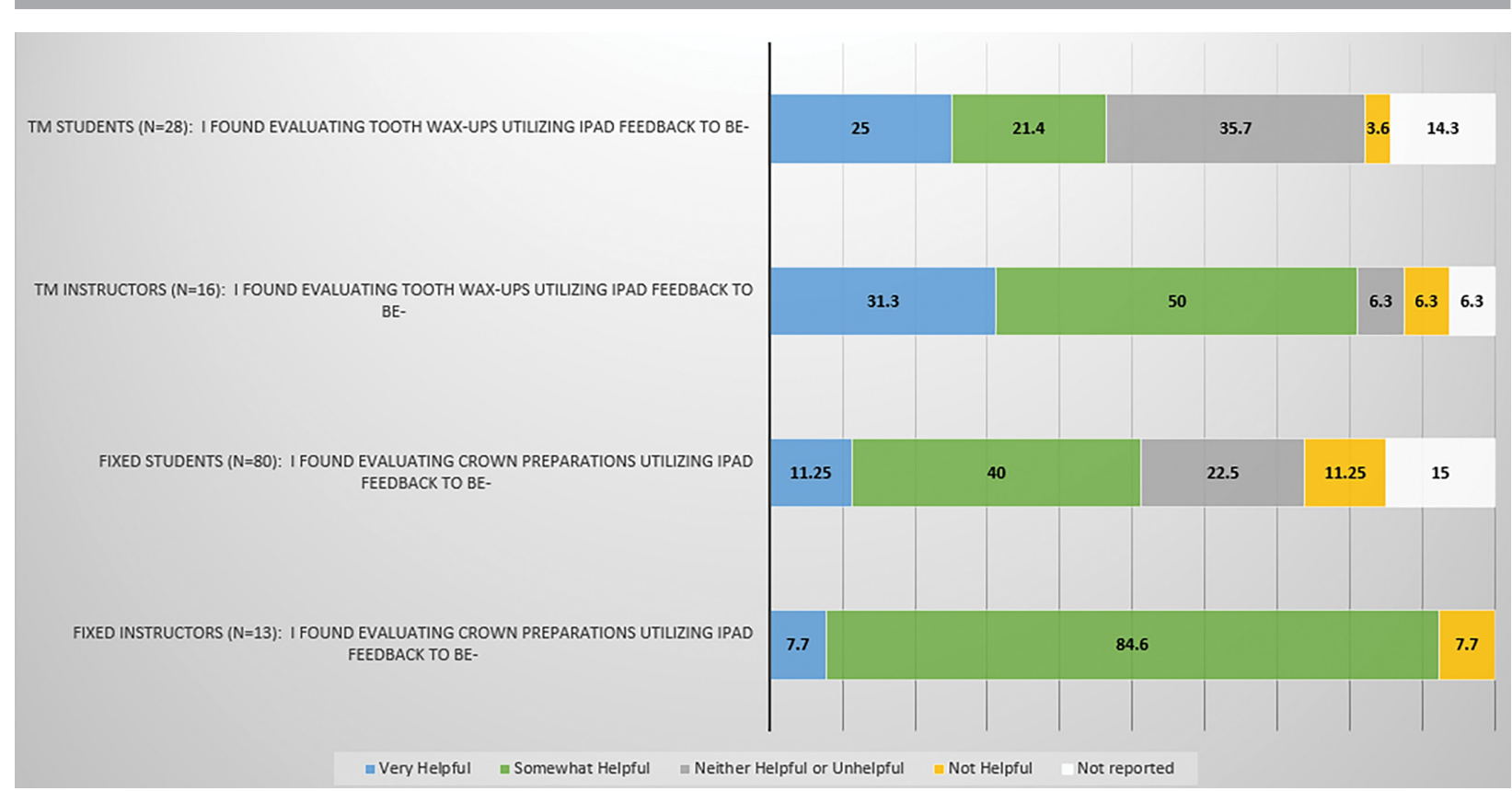

Figure 4. Results of post survey in Tooth Morphology and Fixed Prosthodontics courses, by percentage of responding students and instructors to each item 
difference between the pre and post results was found, with more TM instructor respondents supporting that students appeared to effectively visualize the feedback when using the iPad images opposed to verbal feedback alone $(p=0.008$, using Mantel-Haenszel chi-square tests for ordered categorical data). In the TM course, $53 \%$ of student respondents and $75 \%$ of instructor respondents preferred a combination of verbal and iPad feedback (Figure 5).

In the Fixed course, $51 \%$ of student respondents and $92.3 \%$ of instructor respondents found evaluating crown preparations using iPad feedback to be very helpful or somewhat helpful (Figure 4). Also in the Fixed course, $54 \%$ of these instructors found taking, annotating, and sharing iPad images during student evaluation sessions to be user-friendly or somewhat user-friendly. Similar to the TM cohort, the Fixed students $(51.3 \%)$ and instructors $(77 \%)$ preferred a combination of verbal and iPad image feedback (Figure 5).

\section{Grade Analysis and Student Perceptions}

When we compared three consecutive years of practical examination data using Wilcoxon Signed Rank Tests, there was a significant increase $(\mathrm{p}<0.001)$ in student grades from first exam (pre) compared to the following exam (post) in all three years. Us- ing Wilcoxon Rank Sum Tests, we found the post scores for 2014 and 2015 were significantly lower than in 2016 when the iPad feedback was introduced $(p=0.019$ and $p=0.002$, respectively). Additionally, change scores between the first and second exams in 2014 and 2015 were significantly lower than in 2016 ( $p=0.030$ and $p<0.001$, respectively) (Table 1 ).

In the TM course, $42 \%$ of the student respondents reported that referencing the iPad feedback via Box was very helpful or somewhat helpful. However, there was no significant difference in students' post or change scores for those referencing and not referencing Box following an instructor evaluation session $(p=0.454$ and $p=0.663$, respectively). Additionally, when we compared the TM and the Fixed students, we found that more TM student respondents agreed that referencing the iPad feedback through Box was helpful ( $\mathrm{p}=0.022$, using Mantel-Haenszel chi-square tests for ordered categorical data).

There was a significant increase $(\mathrm{p}<0.001)$ in the Fixed students' grades between the first exam (pre) and the following exam (post) in all three years. The post scores for 2014 and 2015 were significantly higher than in 2016 when the iPad intervention occurred $(p=0.001$ and $p<0.001$, respectively, Wilcoxon Rank Sum tests). The change scores between the first and second exams for 2015 were significantly higher than in 2016 ( $\mathrm{p}=0.019$, Wilcoxon Rank Sum test) (Table 1). Over a fourth (28.7\%) of the Fixed

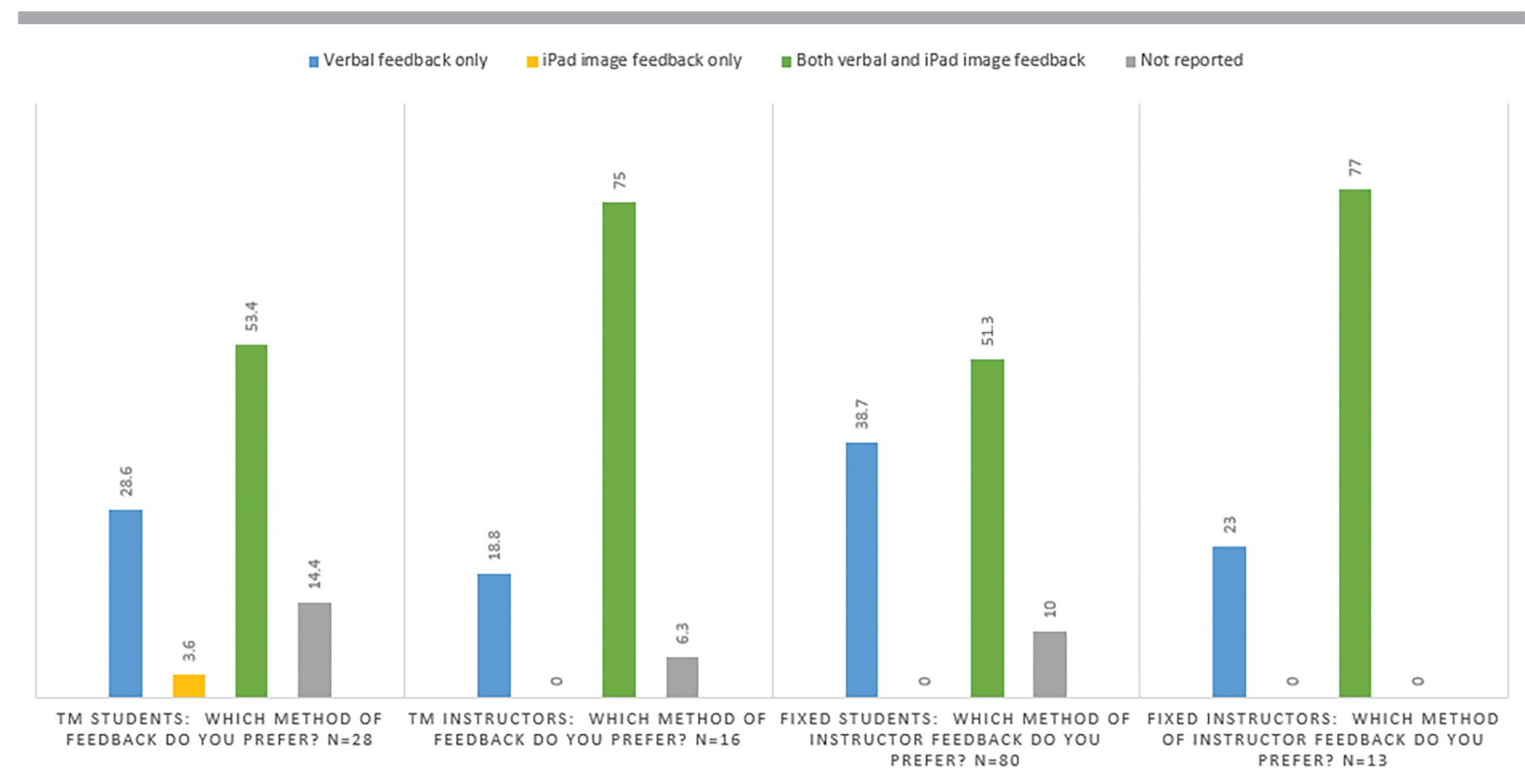

Figure 5. Feedback method preferences in Tooth Morphology (TM) and Fixed Prosthodontics (Fixed) courses, by percentage of responding students and instructors to each item 


\begin{tabular}{|c|c|c|c|}
\hline Course/Exam & Year & $\mathrm{N}$ & Mean (SD) \\
\hline \multicolumn{4}{|l|}{ TM course } \\
\hline Pre & $\begin{array}{l}2014 \\
2015 \\
2016\end{array}$ & $\begin{array}{l}109 \\
107 \\
106\end{array}$ & $\begin{array}{l}15.5(1.8) \\
16.0(1.8) \\
15.3(2.3)\end{array}$ \\
\hline Post & $\begin{array}{c}2014 \\
2015 \\
2016+\end{array}$ & $\begin{array}{l}109 \\
107 \\
106\end{array}$ & $\begin{array}{l}17.1(1.4) \\
17.0(1.3) \\
17.5(1.3)\end{array}$ \\
\hline Change (post-pre) & $\begin{array}{c}2014 \\
2015 \\
2016+\end{array}$ & $\begin{array}{l}109 \\
107 \\
106\end{array}$ & $\begin{array}{c}1.7(1.6) \\
1.0(1.8) \\
2.3^{*}(2.1)\end{array}$ \\
\hline \multicolumn{4}{|l|}{ Fixed course } \\
\hline Pre & $\begin{array}{l}2014 \\
2015 \\
2016\end{array}$ & $\begin{array}{l}104 \\
107 \\
105\end{array}$ & $\begin{array}{l}46.4(4.1) \\
46.6(3.9) \\
45.8(4.2)\end{array}$ \\
\hline Post & $\begin{array}{c}2014 \\
2015 \\
2016+\end{array}$ & $\begin{array}{l}104 \\
107 \\
105\end{array}$ & $\begin{array}{l}57.8(5.5) \\
58.0(5.5) \\
55.4(4.9)\end{array}$ \\
\hline Change (post-pre) & $\begin{array}{c}2014 \\
2015 \\
2016+\end{array}$ & $\begin{array}{l}104 \\
107 \\
105\end{array}$ & $\begin{array}{c}11.3(5.7) \\
11.4(6.4) \\
9.6(6.0)\end{array}$ \\
\hline
\end{tabular}

tWhen iPad feedback was implemented

${ }^{*}$ Change scores for 2014 and 2015 were significantly lower than for 2016 ( $p=0.030$ and $p<0.001$, respectively, Wilcoxon Rank Sum tests).

student respondents found referencing the iPad feedback via Box to be very helpful or somewhat helpful. There was no significant difference in students' post or change scores between those referencing and not referencing Box following an instructor session $(p=0.408$ and $p=0.750$, respectively, Wilcoxon Rank Sum test).

\section{Discussion}

This iPad feedback study examined TM and Fixed student and instructor perceptions of the effectiveness of students' visualizing verbal feedback and student recall ability. The study found while the majority of the TM student respondents believed they could effectively visualize the verbal feedback provided by the instructor, slightly less than half of the TM instructor respondents agreed that students could effectively visualize the feedback. The results from the Fixed course were similar, with the majority of student respondents reporting they could successfully envision the detailed verbal instruction, and half of the instructor respondents reporting the same. This difference between student and instructor observations in both courses could be due to students' overconfidence in their ability to comprehend instructor feedback. Novice learners may not recognize they are incorrectly processing the instructor's feedback. However, instructors are experienced evaluators who are cognizant that development of the novice student's abilities to understand and accurately translate the feedback will take time and repetition. ${ }^{1}$

The student and instructor responses were also compared regarding the ability of students to recall the verbal feedback provided by the instructors. The majority of both TM and Fixed student and instructor respondents agreed that students could recall the instructor feedback following an evaluation session. These findings indicate that although the majority of students tended to perceive their abilities to visualize and recall as satisfactory, the instructors believed the students' visualization process could be improved for better understanding. Furthermore, a small percentage of students reported they struggled with the visualization and recall of verbal feedback. Further research could investigate if there is a correlation between students who cannot visualize and recall the information and unsatisfactory academic performance.

An additional objective for the study was to determine if iPad feedback would enhance the exchange of assessment information. Nearly half of the TM student respondents and the majority of the TM instructor respondents reported on the post survey that evaluating tooth wax-ups with iPad image feedback was very helpful or somewhat helpful. Furthermore, more TM instructor respondents agreed that assessment information was enhanced with iPad images as compared to the effectiveness of verbal feedback alone, and the difference was statistically significant $(p=0.008)$. In the Fixed course, the instructors rated the helpfulness of evaluating crown preparations higher than did the Fixed student respondents, although the difference was not statistically significant. These findings may indicate that instructors' effectiveness in presenting verbal feedback to novice learners could be improved with the addition of a digital teaching tool to reinforce visual concepts.

The study also evaluated student and instructor perceptions regarding instructor feedback method preference. Slightly over half of the TM (53\%) and Fixed (51\%) students and large majorities of the TM (75\%) and Fixed (92.3\%) instructors preferred both verbal and iPad image feedback compared to verbal 
feedback alone, thus indicating an overall positive response. This finding may suggest that traditional feedback could be improved if combined with iPad images, and it may result in earlier acquisition of comprehension and assessment development for students. The Dual-Coding Theory supports these findings by stating that learning is enhanced with use of both visual and verbal cognitive components. ${ }^{17}$ These iPad images provide the instructors with a customized evaluation instrument, which is particularly useful when instructors cannot effectively describe their feedback or when students are unable to comprehend the verbal feedback.

In addition, over half of the TM and Fixed instructor respondents found taking, annotating, and sharing iPad images to be user-friendly or somewhat user-friendly. Several instructors commented that the iPad images can effectively showcase specific areas for improvement, but many commented that the process was time-consuming. If instructors perceive the iPad image process to be difficult or lengthy, they may default to providing only verbal feedback. This possibility may account for the small sample size of TM student respondents on the post survey who reported receiving iPad image feedback. Instructors reported that, with additional training and repeated use, the iPad feedback process could become easier and might result in increased instructor participation.

The final objective of this study was to determine if participating in iPad feedback would improve student grades compared to previous years. For both the TM and Fixed courses, the following factors were constant from 2014 to 2016: grading faculty, course directors, grading rubrics, curricular time, and overall teaching methodology. Also, the class sizes varied slightly by no more than $2 \%$. However, a recognized variable is that the majority of laboratory instructors change each year as fourth-year dental students are selected to serve as instructors in the preclinical laboratories. In both courses, there were significant increases in the overall mean student grades from the first practical exam to the subsequent practical exam. These results demonstrated that, as students acquired fundamental knowledge independently and through instructor feedback, the majority of students improved over time. The findings showed that the change scores between the first and second exams in 2014 and 2015 were significantly lower than in 2016 when iPad feedback intervention occurred, exclusively in the TM course. This outcome may support that iPad feedback could improve student performance in preclinical laboratory courses. Fur- ther research is needed to validate this finding with a larger student sample size. Additionally, the Fixed course results showed no significant change in mean scores following iPad intervention, which may indicate iPad image feedback could be more valuable earlier in the dental curriculum as basic concepts and assessment skills are developing.

We hoped that, by sharing iPad images to Box, students would benefit from post-evaluation reflection, which could help improve their overall recall of the feedback. The study found that more TM student respondents agreed that referencing iPad images in Box was helpful, which was a statistically significant finding $(p=0.022)$ compared to the Fixed student respondents. However, several survey comments from both groups of students suggested they did not find this to be a particularly valuable feature. This observation further corroborates that iPad images may be more useful earlier in the curriculum. There was no statistical difference between students' practical exam scores for those referencing and not referencing Box for both courses. In the Callan et al. study on using CAD/CAM technology as a teaching tool, the authors reported no statistical difference in exam scores between the students who used the CAD/CAM technology and those who did not. ${ }^{9}$ They attributed these results to the lack of use of the CAD/CAM technology. Similarly, the instructors and students in our study did not frequently use the referencing Box feature, which may account for the lack of statistical significance in practical exam scores for those students who did or did not reference Box. Due to the multi-step process of sharing images to Box, the instructors found this feature to be cumbersome. Thus, the results suggest that the additional steps of sharing and accessing the images in Box may not be as helpful as we initially hoped.

We recognize there are further limitations to the use of the iPad image feedback method in preclinical laboratory courses. In both the TM and Fixed courses, instructors commented that the iPad images were more beneficial for certain parameters, such as proper axial alignment. However, the images were not as helpful when conveying three dimensional concepts related to occlusal scheme and clearance. Another limitation is that this study was performed at one institution, and the results may not be applicable to other schools. Future research could measure if iPad image feedback can be applied to other preclinical course disciplines and whether similar results would be obtained upon collaboration with other institutions. 


\section{Conclusion}

Various forms of mobile technology such as iPads are pervasive in today's educational settings, but the documented use of iPads for teaching in dental education is limited. We sought to incorporate a relatively inexpensive and familiar digital technology tool into the IUSD preclinical curriculum to improve students' visualization and comprehension of instructor feedback. Thorough comprehension is necessary for students to accurately translate the given feedback into action. This study found that iPad image feedback in conjunction with verbal feedback resulted in an enhanced exchange of assessment information compared to verbal feedback alone. The outcomes of this study show that academic performance may also be improved with the addition of iPad image feedback, particularly for novice dental students who are in the earliest stages of motor skill development.

\section{Acknowledgments}

The study was funded by a curriculum enhancement grant obtained through the Center for Teaching and Learning at Indiana University Purdue University Indianapolis for the 2016-17 academic year. The authors would like to thank the center for the grant. This project was made possible with support from the IUSD Department of Prosthodontics and Department of Cariology, Operative Dentistry, and Public Health.

\section{REFERENCES}

1. Hauser AM, Bowen DM. Primer on preclinical instruction and evaluation. J Dent Educ 2009;73(3):390-8.

2. Boud DJ, Falchihov N. Quantitative studies of student self-assessment in higher education. Higher Educ 1989; 18:529-49.

3. Mays KA, Levine E. Dental students' self-assessment of operative preparations using CAD/CAM: a preliminary analysis. J Dent Educ 2014;78(12):1673-80.

4. Ramaprasad A. On the definition of feedback. Behav Sci 1983;23:4-13.
5. Sadler DR. Formative assessment and the design of instructional systems. Instruct Sciences 1989;18:119-44.

6. Commission on Dental Accreditation. Accreditation standards for dental education programs. 2018. At: www. ada.org/ /media/CODA/Files/pde.pdf?la=en. Accessed 26 June 2018.

7. Hamil LM, Mennito A, Renne WG, Vuthiganon J. Dental students' opinions of preparation assessment with E4D Compare software versus traditional methods. J Dent Educ 2014;78(10):1424-31.

8. Mays KA, Crisp AH, Vos P. Utilizing CAD/CAM to measure total occlusal convergence of preclinical dental students' crown preparations. J Dent Educ 2015;80(1):100-7.

9. Callan RS, Palladino CL, Furness AR, et al. Effectiveness and feasibility of utilizing E4D technology as a teaching tool in a preclinical dental education environment. J Dent Educ 2014;78(10):1416-23.

10. Park CF, Sheinbaum JM, Tamada Y, et al. Dental students' perceptions of digital assessment software for preclinical tooth preparation exercises. J Dent Educ 2017;81(5): 597-603.

11. Alyahya S, Gall JE. iPads in education: a qualitative study of students' attitudes and experiences. Paper presented at World Conference on Educational Multimedia, Hypermedia, and Telecommunications, 2012.

12. McCombs S, Liu Y. Channeling the channel: can iPad meet the need of today's m-learner? Presentation at Society for Information Technology \& Teacher Education International Conference, 2011.

13. Stein CD, Eisenberg ES, O'Donnell JA, Spallek H. What dental educators need to understand about emerging technologies to incorporate them effectively into the education process. J Dent Educ 2013;78(4):520-9.

14. Gadbury-Amyot CC, Purk JH, Williams BJ, Van Ness CJ. Using tablet technology and instructional videos to enhance preclinical dental laboratory learning. J Dent Educ 2013;78(2):250-8.

15. Aragon CE, Zibrowski EM. Does exposure to a procedural video enhance preclinical dental student performance in fixed prosthodontics? J Dent Educ 2007;72(1):67-71.

16. Shah DY, Dadpe AM, Kalra DD, Garcha VP. Videotaped feedback method to enhance learning in preclinical operative dentistry: an experimental study. J Dent Educ 2015;79(12):1461-6.

17. Clark JM, Paivio A. Dual coding theory and education. Educ Psychol Rev 1991;3:149-210. 